\title{
ABSOLUTE CONTINUITY OF CERTAIN UNITARY AND HALF-SCATTERING OPERATORS
}

\author{
C. R. PUTNAM ${ }^{1}$
}

1. The theorem. Let $A$ be a self-adjoint operator, bounded from below, and $D$ a bounded, non-negative self-adjoint operator on a Hilbert space $\mathfrak{S}$ for which the set

$$
\mathfrak{D}_{A} \cap \Re_{D^{1 / 2}} \text { is dense. }
$$

Suppose that $A+D$ is unitarily equivalent to $A$ and that $U$ is any unitary operator effecting this equivalence, thus

$$
A+D=U A U^{*} \text {. }
$$

Then $U$ is absolutely continuous, that is, if $U$ has the spectral resolution

$$
U=\int_{0}^{2 \pi} e^{i \lambda} d E(\lambda)
$$

and if $x$ is an arbitrary element of the Hilbert space, then $\|E(\lambda) x\|^{2}$ is an absolutely continuous function of $\lambda$.

If $A$ is bounded, then $\mathfrak{D}_{A}=\mathfrak{S}$ and (1) reduces to the assumption that 0 cannot be in the point spectrum of $D$. In this case, the assertion of the theorem was proved in [2]. In [3], there were obtained lower estimates for the measure of the spectrum of $U$ both when $A$ was bounded and also in the case when $A$ was supposed only halfbounded. It will be shown in the present paper that the methods used in this latter case will also yield the absolute continuity of $U$ under the conditions specified in the theorem.

An application to half-scattering operators will be given in $\$ 3$.

2. Proof of the theorem. Since (1) and (2) hold if $A$ is replaced by $A+c I$, where $c=$ const., it is clear that there is no loss of generality in assuming that

$$
A \geqq 0 \text {. }
$$

If $f(\lambda)$ is a real-valued function of period $2 \pi$ with a continuous first derivative and having the Fourier series

(5) $f(\lambda)=\sum_{k=-\infty}^{\infty} c_{k} e^{i k \lambda}, \quad c_{k}=(2 \pi)^{-1} \int_{0}^{2 \pi} f(\lambda) e^{-i k \lambda} d \lambda, \quad c_{-k}=\bar{c}_{k}$,

Received by the editors November 14, 1961.

1 This research was supported by the Air Force Office of Scientific Research. 
then it follows from (3) and (5) that

$$
D^{1 / 2} \int_{0}^{2 \pi} f(\lambda) d E(\lambda)=c_{0} D^{1 / 2}+\sum_{k=1}^{\infty} c_{k} D^{1 / 2} U^{k}+\sum_{k=1}^{\infty} \bar{c}_{k} D^{1 / 2} U^{* k} .^{2}
$$

Next, let $y$ be in $\mathfrak{D}_{A} \cap \Re_{D^{1 / 2}}$, so that $y=D^{1 / 2} x$ is in $\mathfrak{D}_{A}$. It follows from (6) that

$$
\begin{aligned}
\left(x, D^{1 / 2} \int_{0}^{2 \pi} f(\lambda) d E(\lambda) y\right) & \\
= & \left(x, c_{0} D^{1 / 2} y\right)+2 \operatorname{Re}\left(x, \sum_{k=1}^{\infty} c_{k} D^{1 / 2} U^{k} y\right) .
\end{aligned}
$$

Since $(\operatorname{Re}(\cdots))^{2} \leqq\|x\|^{2}\left(\sum_{k=1}^{\infty}\left|c_{k}\right|^{2}\right)\left(\sum_{k=1}^{\infty}\left\|D^{1 / 2} U^{k} y\right\|^{2}\right)$, an application of the Schwarz inequality to $(7)$ yields

$$
\begin{aligned}
\left(\int_{0}^{2 \pi} f(\lambda) d\|E(\lambda) y\|^{2}\right)^{2} & \\
& \leqq 2\left[\left|c_{0}\right|^{2}\|y\|^{4}+4\|x\|^{2}\left(\sum_{k=1}^{\infty}\left|c_{k}\right|^{2}\right)\left(\sum_{k=1}^{\infty}\left\|D^{1 / 2} U^{k} y\right\|^{2}\right)\right] .
\end{aligned}
$$

But (2) implies that for $n=1,2, \cdots, \sum_{k=1}^{n} U^{* k} D U^{k}=A-U^{*_{n}} A U^{n}$ $\leqq A$, the inequality by (4), and so $\sum_{k=1}^{\infty}\left\|D^{1 / 2} U^{k} y\right\|^{2} \leqq(A y, y)$.

Relation (8) and the Parseval relation $(2 \pi)^{-1} \int_{0}^{2 \pi} f^{2}(\lambda) d \lambda=\left|c_{0}\right|^{2}$ $+2 \sum_{k=1}^{\infty}\left|c_{k}\right|^{2}$ now imply

$$
\left(\int_{0}^{2 \pi} f(\lambda) d\|E(\lambda) y\|^{2}\right)^{2} \leqq C(x) \int_{0}^{2 \pi} f^{2}(\lambda) d \lambda,
$$

where $C(x)$ is a number which depends on $x$ (and $y=D^{1 / 2} x$ ) but not on the choice of $f(\lambda)$. Since (9) holds for every smooth function on $[0,2 \pi]$ satisfying $f(0)=f(2 \pi)$ and since ordinary Lebesgue measure is absolutely continuous, it follows by a standard argument (involving approximations of characteristic functions of intervals by smooth functions $f(\lambda)$ satisfying $f(0)=f(2 \pi)$ ) that $\left.\|E(\lambda) y\|\right|^{2}$ is also absolutely continuous. It then follows from (1) that $\|E(\lambda) x\|^{2}$ is absolutely continuous for all $x$ in the Hilbert space and the proof of the theorem is complete.

3. Half-scattering operators. Let $A$ denote the quantum mechanical (half-bounded) energy operator $-d^{2} / d x^{2}$ on the Hilbert space

'Since $f(\lambda)$ has a continuous derivative, then $\left\|\sum_{k-\infty}^{\infty} c_{k} D^{1 / 2} U^{k}\right\|$ $\leqq\left(\sum_{k-\infty}^{\infty}\left|c_{k}\right|\right)\left\|D^{1 / 2}\right\|<\infty$, and so the summations of (6) converge in the uniform norm topology. The author is indebted to the referee for this observation. 
$L^{2}(-\infty, \infty)$ and $D$ be a perturbation potential $V(x)$, where $V(x)$ is continuous and satisfies

$$
0 \leqq V(x)<\text { const., } \quad-\infty<x<\infty,
$$

and

$$
\int_{-\infty}^{\infty} V(x) d x<\infty
$$

Then $A$ and $A+D$ are absolutely continuous and each has the halfline $0 \leqq \lambda<\infty$ as spectrum (cf. [4] and the references there to Weyl, Kodaira, Titchmarsh). Moreover, it follows from results of Kuroda [1] (cf. [4]) that the half-scattering operators

(12) $U_{+}=\lim _{t \rightarrow \infty} U_{t}$ and $U_{-}=\lim _{t \rightarrow-\infty} U_{t}$, where $U_{t}=e^{i t(A+D)} e^{-i t A}$,

exist as strong limits satisfying (2). As a consequence of the theorem of the present paper, it follows that if, in addition to (10) and (11), $V(x)$ satisfies

$$
V(x)>0 \text { almost everywhere on }(-\infty, \infty),
$$

then the half-scattering operators $U_{+}$and $U_{-}$of (12) are absolutely continuous. In fact, (10) implies that $D=V(x)$ is bounded and nonnegative while (13) implies (1).

It can be noted that if, in addition to (10), (11) and (13), also

$$
\lim \inf (b-a)^{-3} \int_{a}^{b} V^{-1}(x) d x=0, \text { as } \quad b-a \rightarrow \infty,
$$

is assumed, then, as was shown in [4], the (absolutely continuous) spectrum of each of the operators $U_{+}$and $U_{-}$must be the entire unit circle $|z|=1$.

\section{REFERENCES}

1. S. T. Kuroda, On the existence and the unitary property of the scattering operator, Nuovo Cimento (10) 12 (1959), 431-454.

2. C. R. Putnam, On differences of unitarily equivalent self-adjoint operators, Proc. Glasgow Math. Assoc. 4 (1960), 103-107.

3. - Commutators, perturbations, and unitary spectra, Acta Math. 106 (1961), $215-322$.

4. - On the spectra of unitary half-scattering operators, Quart. J. Appl. Math. 20 (1962), 85-88.

Purdue University 